\title{
PRELIMINARY RESULTS FROM A SUPERCONDUCTING PHOTOCATHODE SAMPLE CAVITY*
}

\author{
P. Kneisel ${ }^{\#}$, TJNAF, Newport News, USA \\ J. Sekutowicz, DESY, 22603 Hamburg,Germany \\ R. Lefferts and A. Lipski, State University of New York at Stony Brook, USA
}

\begin{abstract}
Pure niobium has been proposed as a photocathode material to extract directly photo-currents from the surface of a RF-gun cavity [1]. However, the quantum efficiency of niobium is $\sim 3 \cdot 10^{-4}$, whereas electro- or vacuum deposited lead has a $\sim 10$ times higher quantum efficiency. We have designed and tested a photo-injector niobium cavity, which can be used to insert photocathodes made of different materials in the high electric field region of the cavity. Experiments have been conducted with niobium and lead, which show that neither the Q- values of the cavity nor the obtainable surface fields are significantly lowered. This paper reports about the results from these tests.
\end{abstract}

\section{INTRODUCTION}

The interest in superconducting photo-injectors has increased in recent years due to the demand for high quality beams, e.g. high brightness, in FEL light sources or energy recovery linacs. High brightness is usually achieved by locating the photo-cathode in a high electric field; high average currents for the accelerator are obtained by $\mathrm{cw}$ operation of the injector; both requirements are achievable with superconducting cavities.

The first proposal for a superconducting photo-injector was published in 1988 [2] and first experiments were done at the University of Wuppertal [3]. At the FZ Rossendorf a SRF gun with a half-cell, $1300 \mathrm{MHz}$ cavity was developed and in a proof-of-principle experiment the feasibility of such a device was successfully demonstrated [4]. A $3 \frac{1}{2}$ cell SRF photo-injector for the ELBE linac is now under construction [5].

One of the problems associated with the use of a "conventional" photo-cathode such as CsTe in a superconducting cavity is the possibility of contamination of the sensitive niobium surfaces by the cathode material. This will result in performance degradations of the SRF cavity both in Q-value and in achievable gradient. Therefore, at BNL [6] the niobium of the cavity was for some time considered as the photo-cathode. However, the quantum efficiency of niobium is rather small and powerful lasers are needed to extract significant current

\footnotetext{
*Supported in part by DOE contract DE-AC0584ER40150

"Kneisel@jlab.org
}

out of the material. Therefore, the BNL group has proposed and is developing a high current photo-cathode based on a sandwiched device consisting of a conventional photo-material such as CsTe and a specially prepared diamond window [7]. Primary electrons are

generated at the photocathode and are accelerated to a few $\mathrm{keV}$, when they strike the diamond window. An amplification of the current by two orders of magnitude is expected due to the high secondary electron yield of the diamond. Such a photo-cathode has to be electrically/rf wise isolated from the superconducting cavity - as in the case of the Rossendorf gun - in order to maintain the high Q-value and high gradients of the superconducting cavity. This complicates somewhat a design.

One of the authors [JS] proposed two years ago [8] to use lead as the photocathode material since it has much higher quantum efficiency than niobium and it is also a superconducting material. In addition it could be directly used as coating on the niobium surface. Such a system - if successfully implemented - would significantly simplify a SRF based photo-injector. After the successful verification of a 10 times higher quantum efficiency of electro-deposited lead [9] compared to niobium, we have investigated the possibility of a lead-based photo-cathode in a niobium cavity and in the following we report about our failures and successes.

\section{THE CAVITY}

There are two choice how one can implement the lead photo-cathode in a niobium cavity: one can either directly deposit a lead spot onto the end wall of the cavity through the beam pipe or one can design a cavity, which provides for the insertion/assembly of a removable lead covered photocathode sample. We have chosen the second approach and the cavity was designed as a test vehicle to evaluate several approaches for photocathode layout with the main interest of exploring the achievable $\mathrm{Q}$ - values and surface electric fields; no attempt has been made in the design to actually use this cavity as a photo-injector.

The cavity as shown in Figure 1 is a half cell of the original CEBAF shape with an endplate This endplate has a tapered hole on axis of $10 \mathrm{~mm}$ diameter and a cut-off tube welded on its backside. This tube is being used to attach the photocathode samples as discussed below. No stiffeners have been added to the endplate to minimize the pressure sensitivity of the cavity. A side port in the beam tube is used for the transmitted signal. The cavity is resonating at $1420 \mathrm{MHz}$. 

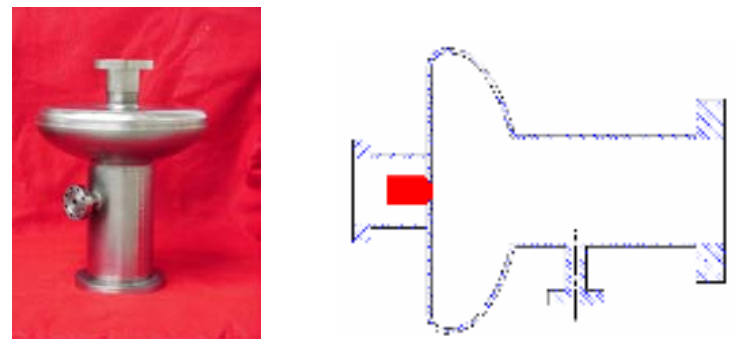

Figure 1: Photocathode cavity, the photocathode insert is shown in the right sketch

The field distribution and the cavity parameters have been calculated with a 2D electromagnetic code [10] for several cases: (i) the sample being inserted from the back and flush with the wall of the endplate, (ii) the sample retracted $\sim 200 \mu \mathrm{m}$ into the cut-off tube and (iii) a "mushroom sample" as shown in figure 3 being assembled to the inside of the endplate. Results of the calculation for the case of the flush sample are shown in Figure 2. Parameters are summarized in Table 1 for 1 Joule of stored energy for all three cases. Both mushroomgeometry and the retracted geometry show strong field enhancement due to the small curvatures exposed to electric fields.
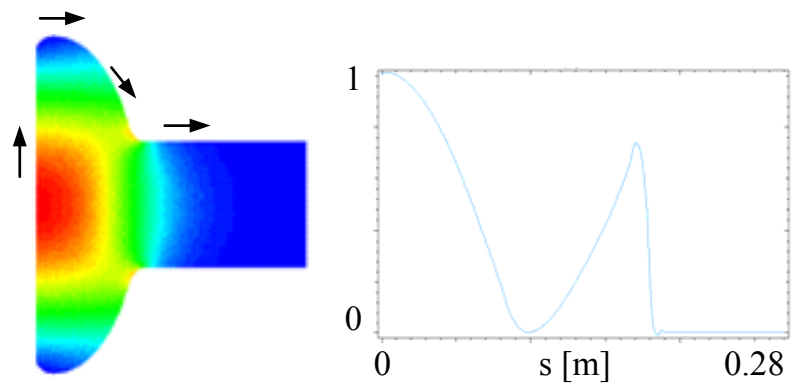

Figure 2: Normalized electric field distribution in the 1.42 $\mathrm{GHz}$ cavity and along the cavity surface (as indicated by arrows).

Table 1: Parameters for different photo cathodes

\begin{tabular}{|c|c|c|}
\hline Geometry & $\begin{array}{c}\text { Geometry } \\
\text { factor }\end{array}$ & $\begin{array}{c}\mathrm{E}_{\text {peak }} \text { at } \mathrm{W}=1 \mathrm{~J} \\
{[\mathrm{MV} / \mathrm{m}]}\end{array}$ \\
\hline Flush with endplate & 176.9 & 25.0 \\
\hline$-200 \mu \mathrm{m}$ & 177.0 & 41.7 \\
\hline Mushroom & 176.8 & 61.5 \\
\hline
\end{tabular}

\section{PHOTOCATHODE GEOMETRIES, ASSEMBLIES AND RESULTS}

Various cathode geometries as shown in figure 3 were tried out on the cavity:

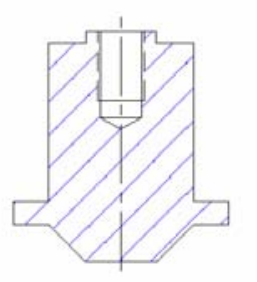

Plug \#2

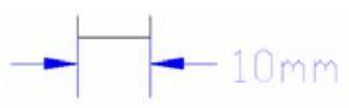

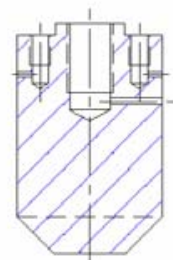

Plug \#1

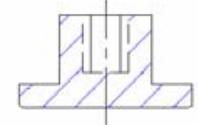

"Mushroom"

Figure 3: Cathode geometries

\section{Plug \#1}

Plug \#1 was assembled from the outside on an axially variable bellows arrangement. The idea was to get information in the same test on the performance of the cavity with the plug retracted and then inserted in the hole. As it turned out this was not a good arrangement. In the retracted position the field leaking into the cut-off tube was not attenuated, because the plug and the cut-off tube formed a coaxial line. Propagating wave to the lossy parts of the variable bellows arrangement, spoiling the Q-value of the cavity. In the inserted position the plug was not well enough aligned to make good contact with the tapered cavity endplate and also unexpected high losses occurred.

\section{The "mushroom"}

The "mushroom" - type cathode was inserted from the inside into the cavity and sealed with thin indium wire to the endplate. This configuration worked well for a few tests, however the vacuum sealing was very difficult to achieve without squeezing indium into the cavity space. A result of a successful test is shown in Figure 4.

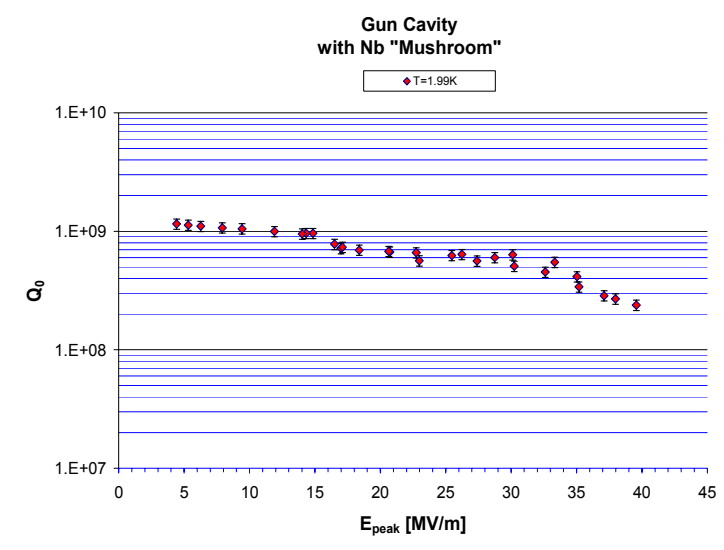

Figure 4: Niobium "Mushroom" photocathode

\section{Plug \#2}

The most successful assembly was with Plug \#2, which is a modification of plug \#1 with a small collar, allowing 
to seal the cathode against the cavity endplate from the with Indium from the outside. This configuration has the additional advantage that the danger of cavity contamination is significantly reduced compared to the "mushroom" cathode. Figure 5 shows the results from a niobium cathode (no coating) and with the cathode coated with a 2-3 $\mu \mathrm{m}$ thick lead spot of $10 \mathrm{~mm}$ diameter. Between the two tests the cavity had been buffered chemically polished by $\sim 10 \mu \mathrm{m}$, which is the standard treatment between tests after an initial removal of $\sim 100$ $\mu \mathrm{m}$ for the baseline tests.

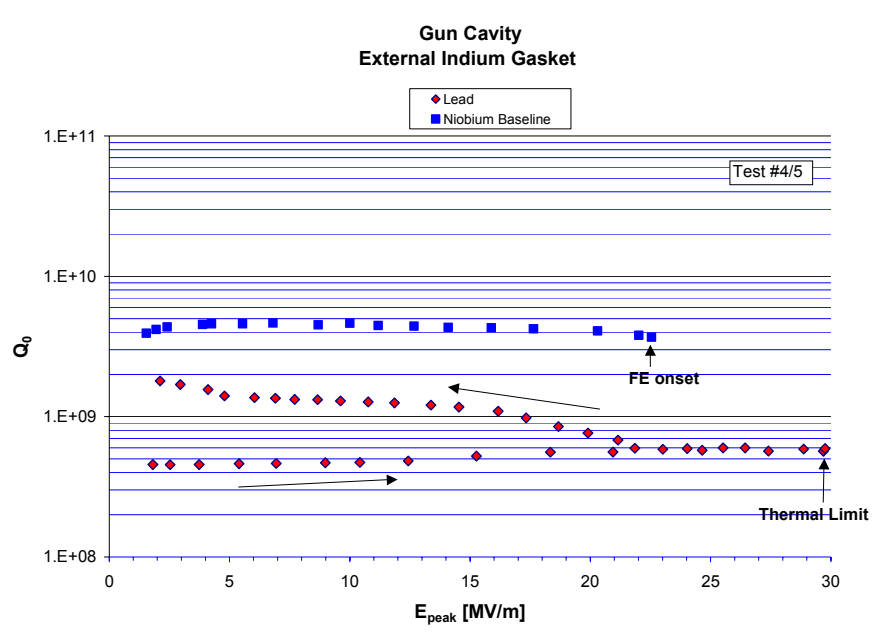

Figure 5: Niobium and lead plated cathode inserts; the lead cathode processed with increasing field and higher Q-values resulted from the "high field cleaning"; at $\sim 30$ $\mathrm{MV} / \mathrm{m}$ the cavity/cathode was limited by insufficient cooling.

\section{FUTURE PLANS}

We believe that the cooling of the cathode insert can be improved by drilling additional channels into the back of the cathode plug and that it might be possible to achieve higher surface fields. Also, we want to explore the performance of electro-deposited lead.

As a next step we want to fabricate a 1.6-cell gun cavity of the LL-ILC shape at $1.3 \mathrm{GHz}$. The so called split superconducting injector at this frequency was proposed in ref [12]. We plan to test this device under $2 \mathrm{~K}$ conditions at BNL.

\section{SUMMARY}

A half cell prototype niobium cavity has been used to explore the possibility of inserting photo cathodes of different configurations. The results show that one can achieve reasonable peak surface fields and Q-values with small lead layers, which are deposited on a niobium substrate. Further improvements seem possible, if the heat transfer conditions from the cathode to the helium bath are improved.

\section{ACKNOWLEDGEMENT}

We would like to thank our colleagues from Jefferson Lab - L. Turlington, B. Manus, G. Slack, S. Manning and P. Kushnick - for their support during this investigation.

\section{REFERENCES}

[1] I. Ben-Zvi et al.; this conference.

[2] H. Piel et al.; FEL '88, Jerusalem, Israel (1988).

[3] A. Michalke et al.; EPAC '92, Berlin, Germany (1992), p.1014.

[4] D. Janssen et al.;Nucl.Instr, and Meth. A507 (2002). 314.

[5] J. Teichert et al.; EPAC 2004, Lucerne, Switzerland (2004), 333.

[6] T. Srinivan-Rao et al.;PAC2003,Portland (2003).

[7] I. Ben-Zvi et al.; BNL Report C-A/AP/\#149

[8] J. Sekutowicz et al., TESLA-FEL report 2004-03 and PRST-AB 2005.

[9] J. Smedley et al.; EPAC 2004, Lucerne, Switzerland (2004), 1126.

[10] J. Sekutowicz, Proceedings of Linear Accelerator Conference, Tsukuba, Japan,1994

[11] M. Ferrario et al.; EPAC 2004, Lucerne, Switzerland (2004), 402.

[12] J. Sekutowicz et al, to be published in PRST 